\title{
Neural tube-ectoderm interactions are required for trigeminal placode
}

\section{formation}

\author{
Michael R. Stark ${ }^{1,2}$, John Sechrist ${ }^{1}$, Marianne Bronner-Fraser ${ }^{1, *}$ and Christophe Marcelle ${ }^{1}$ \\ ${ }^{1}$ Division of Biology 139-74, California Institute of Technology, Pasadena, CA 91125, USA \\ 2Department of Developmental and Cell Biology, University of California, Irvine, CA 92697, USA \\ *Author for correspondence
}

\section{SUMMARY}

Cranial sensory ganglia in vertebrates develop from the ectodermal placodes, the neural crest, or both. Although much is known about the neural crest contribution to cranial ganglia, relatively little is known about how placode cells form, invaginate and migrate to their targets. Here, we identify Pax-3 as a molecular marker for placode cells that contribute to the ophthalmic branch of the trigeminal ganglion and use it, in conjunction with DiI labeling of the surface ectoderm, to analyze some of the mechanisms underlying placode development. Pax -3 expression in the ophthalmic placode is observed as early as the 4-somite stage in a narrow band of ectoderm contiguous to the midbrain neural folds. Its expression broadens to a patch of ectoderm adjacent to the midbrain and the rostral hindbrain at the 8- to 10-somite stage. Invagination of the first Pax-3-positive cells begins at the 13-somite stage. Placodal invagination continues through the 35-somite stage, by which time condensation of the trigeminal ganglion has begun. To challenge the normal tissue interactions leading to placode formation, we ablated the cranial neural crest cells or implanted barriers between the neural tube and the ectoderm. Our results demonstrate that, although the presence of neural crest cells is not mandatory for Pax -3 expression in the forming placode, a diffusible signal from the neuroectoderm is required for induction and/or maintenance of the ophthalmic placode.

Key words: placode, cranial ganglion, neural crest, Pax-3, FREK, chick

\section{INTRODUCTION}

The trigeminal ganglion, which provides sensation for much of the face, has served as a good experimental system for investigating the development of peripheral ganglia because of its size and accessibility (reviewed in Davies, 1988). Trigeminal sensory neurons originate from two distinct embryonic cell populations: the neural crest and the ectodermal placodes (Yntema, 1942; Hamburger, 1961; Noden, 1978; Narayanan and Narayanan, 1980; Ayer-LeLièvre and Le Douarin, 1982; D'Amico-Martel and Noden, 1980, 1983; reviewed by Noden, 1993). The cranial neural crest population exits from the dorsal neural tube and migrates under the head ectoderm. In addition to contributing neurons and glia to cranial ganglia, neural crest cells form connective tissue and bones of the face and skull (reviewed in Le Douarin, 1982). The cranial sensory placode population undergoes an epithelial-mesenchymal transition from a thickened ectodermal epithelium; these cells then invaginate, migrate, condense and differentiate into neurons, receptors and some support cells of the peripheral nervous system (reviewed by Webb and Noden, 1993).

Unlike most placodes, the trigeminal placode is not morphologically distinct from the surrounding ectoderm. As a consequence, most information about its development comes from observations of placode cells during their migration and gangliogenesis. The trigeminal ganglion comprises two lobes: the ophthalmic lobe and the maxillomandibular lobe. Both receive contributions from ectodermal placodes and neural crest cells. In amphibians, the two lobes remain separate or fuse secondarily during development, suggesting that they are embryologically and evolutionarily distinct (Hamburger, 1961; Northcutt and Brandle, 1995). Histological analyses in the mouse and chick indicate that placode cells leave the ectodermal layer by breaking through the basal lamina as individuals or small clusters of cells (Hamburger, 1961; D'Amico-Martel and Noden, 1983; Nichols, 1986; reviewed by Webb and Noden, 1993). They then migrate to the distal regions of the trigeminal ganglion. Trigeminal placode cells become immunoreactive for neuronal markers and exit the cell cycle early in their development (Moody et al., 1989; D'AmicoMartel and Noden, 1980). In contrast, the neural crest component of the trigeminal ganglion only expresses neuronal markers after condensation. If placode cells fail to invaginate, they can form ectopic ganglia in the surface ectoderm (Kuratani and Hirano, 1990), suggesting that migration and interaction with neural crest cells are not necessary for their neuronal differentiation.

Molecular markers of undifferentiated placodal epithelia have not previously been described (Webb and Noden, 1993), making it difficult to characterize the induction and cell specification of this cranial placode. In this study, we have used the transcription factor Pax-3 and the FGF receptor FREK as 
molecular markers to analyze the spatiotemporal development of the ophthalmic placode. These markers were used in combination with DiI labeling and neurofilament immunocytochemistry to characterize the early specification of the ophthalmic placode, as well as to analyze the neuronal differentiation and subsequent gangliogenesis of placode cells contributing to the trigeminal ganglion. We then investigated the possible mechanisms of placode induction experimentally and found that interactions between the neuroectoderm and surface ectoderm, but not the neural crest, are required for normal ophthalmic placode formation.

\section{MATERIALS AND METHODS}

\section{Cloning of quail Pax-3}

To isolate a Pax-3 probe for use in whole-mount in situ hybridizations, we used a fragment of the chick Pax-3 cDNA (Goulding et al., 1993) to screen a 4-day-old quail whole embryo cDNA library (InVitrogen). We isolated four clones, the longest of which spans $3080 \mathrm{bp}$. Comparison of its sequence (GenBank accession number AF000673) to the mouse Pax-3 cDNA revealed that the quail clone spans $1442 \mathrm{bp}$ of coding sequence and around $1.6 \mathrm{~kb}$ of $3^{\prime}$ untranslated region, and that it is missing the nucleic acid sequence corresponding to the first 5 amino acids of the Pax-3 polypeptide (Fig. 1). Comparison of the deduced amino acid sequences of the quail Pax-3 to the mouse Pax3 indicates that they are $93 \%$ similar, while their nucleic acid identity is $46 \%$ ( $81 \%$ in the coding region). Sequencing was performed using an ALF automated sequencer (Pharmacia) as well as the standard dideoxy chain termination method with radiolabeled nucleotides (Sanger et al., 1977).

\section{In situ hybridization}

Pax-3 digoxigenin-labeled RNA probes were synthesized and used for whole-mount in situ hybridization on fixed quail and chick embryos as described by Wilkinson (1992) and Henrique et al. (1995). Embryos were embedded in gelatin and prepared for cryostat sectioning as described by Sechrist et al. (1995); 10-20 $\mu \mathrm{m}$ sections were mounted on subbed slides.

\section{Dil labeling}

To label the surface ectoderm, but not the neural tube or neural crest, whole ectoderm DiI labeling was performed following closure of the cranial neural tube (i.e. after the 12-somite stage) as previously described (Sechrist et al., 1995). Briefly, a small hole was made in the vitelline membrane above the embryo, through which the DiI/sucrose solution was applied (Cell Tracker DiI, Molecular Probes). After allowing them to develop to the desired stage, embryos were collected, fixed in $4 \%$ paraformaldehyde and prepared for cryostat sectioning.

\section{Ablations and barrier placements}

Fine glass needles were used to remove the dorsal third of the neural tube as described previously (Sechrist et al., 1995) or the surface ectoderm. For placement of barriers, glass needles were used to cut a slit between the ectoderm and the neural folds and the barriers were inserted with fine forceps. $7.5 \mu \mathrm{m}$ thick tantalum foil (Goodfellow \#TA000280) was cut into pieces of approximately $250 \times 350 \mu \mathrm{m}$ and shaped with fine forceps. Polycarbonate membranes (Osmotics) with pore sizes of $0.1 \mu \mathrm{m}$ or $0.8 \mu \mathrm{m}$ were cut into similar sized pieces. One day after barrier insertion, embryos were collected for in situ hybridization analysis.

\section{Neurofilament immunoreactivity}

Sections of DiI-labeled embryos were stained with a neurofilament antibody (kindly provided by Dr Virginia Lee) as described previously (Sechrist et al., 1993).

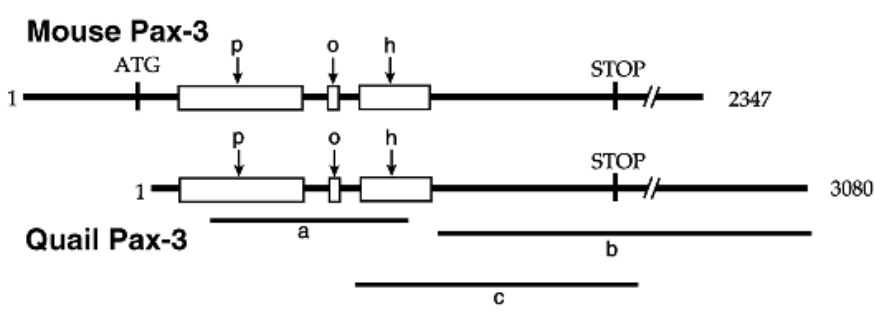

Fig. 1. Quail Pax-3 gene. Comparison of the mouse (Goulding et al., 1991) and quail Pax-3 genes showing regions of the paired domain (p), the octapeptide (o) sequence and the homeodomain (h). The quail gene was isolated from a 4-day-old quail library using a chick probe (a) (Chalepakis et al., 1993). In situ hybridizations perfomed in the current study used probes (b and c) synthesized from two deletion fragments of the quail Pax-3 gene. GenBank accession number, AF000673.

\section{RESULTS}

\section{Analysis of trigeminal placode development using ectodermal Dil-labeling}

To examine the contribution of neural crest and placode cells to the trigeminal ganglion, we selectively labeled the surface ectoderm with DiI. Surface labeling was performed after neural tube closure to avoid labeling the neural tube/neural crest. Because placode cells are the only surface ectoderm cells to invaginate, any DiI-labeled cells within the mesenchyme derive from the ectodermal placodes. When embryos were labeled at the 15 somite stage and collected at the 19-somite stage, we observed several individual, DiI-positive cells within the mesenchyme adjacent to the midbrain and rostral hindbrain (Fig. 2A). In slightly older embryos, additional DiI-positive placode cells were visible in this region (Fig. 2B); placode-derived cells contributing to the ophthalmic branch ultimately aligned in a long array extending from the rostral hindbrain up to the caudal portion of the developing eye (Fig. 2C). As the ganglion became morphologically distinct from the surrounding mesenchyme (30- to 35-somite stage), placode cells could be observed in the distal regions of each lobe of the trigeminal ganglion. In contrast, unlabeled neural crest cells resided in the more proximal regions of the ganglion (Fig. 2D). These results are in good agreement with previous studies using alternative labeling techniques (D’Amico-Martel and Noden, 1983).

\section{Pax-3 and FREK are molecular markers of the ophthalmic trigeminal placode and ganglion}

Although whole ectoderm DiI labeling provides an efficient means to analyze placode cell invagination and gangliogenesis, this technique is not suitable to study placode development prior to cell invagination and does not provide a means by which to investigate placode induction and specification. Moreover, using this technique, we cannot examine placode cells that may invaginate prior to neural tube closure. We therefore sought to identify molecular markers of the differentiating trigeminal placode.

We found that the transcription factor Pax-3 (Goulding et al., 1991) and the FGF receptor FREK (Marcelle et al., 1994) are early markers of the avian ophthalmic placode and used these to analyze the early specification of placodal cells. The earliest indication of ectodermal Pax-3 expression in the head occurs 


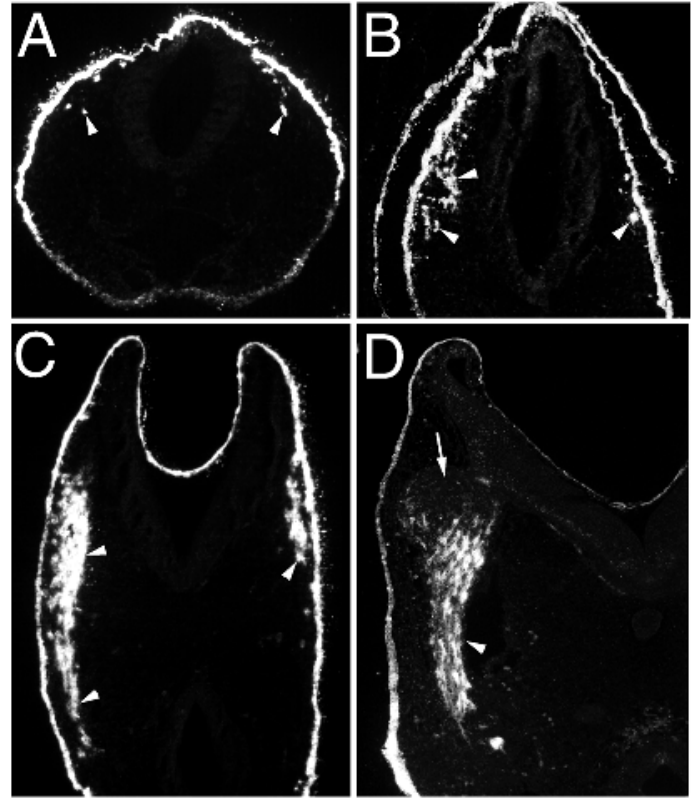

Fig. 2. DiI labeling of the surface ectoderm reveals placode cell migration and subsequent ganglion contributions. Sections through the trigeminal region of embryos labeled with DiI: (A) at the 15somite stage and collected at 19-somite stage; (B) at 12-somite stage and collected at 21-somite stage; (C) at 15-somite stage and collected at 29-somite stage; (D) at 19-somite stage and collected at 34-somite stage. Since only the surface ectoderm was labeled, all internal DiIpositive cells are placode derived. Arrowheads indicate placodederived cells (A-D). The neural crest component of the trigeminal ganglion remains unlabeled $(\mathrm{D}$, arrow).

at the 4-somite stage, at which time Pax-3-positive ectodermal cells are contiguous to Pax-3-positive cells in the dorsal neural tube and neural folds (Fig. 3A,B). Ectodermal Pax-3 expression expands laterally and increases in intensity as development proceeds. By the 6-somite stage (Fig. 3C,D), Pax3 is clearly visible in the dorsolateral ectoderm overlying the head mesenchyme at the level of the midbrain and rostral hindbrain. As neural crest cells initiate migration at the 7- to 8-somite stage, Pax-3 expression is detected in a broadening area of surface ectoderm adjacent to the presumptive midbrain and rostral hindbrain (Fig. 3E,F). In 12- to 16-somite-stage embryos, the ectodermal Pax-3 expression domain becomes restricted to a band of intensely labeled cells extending from the rostral hindbrain towards the dorsal eye region (Fig. 3G, H).

Pax-3-positive placode cells begin to enter the mesenchyme as early as the 13-somite stage (Fig. 4A), with the majority of cells invaginating and becoming migratory in 18- to 26-somitestage embryos. As previously described (reviewed by Noden, 1993), placode cells appear to exit the ectoderm individually or as small clusters (Fig. 3H, arrowhead; Fig. 4A,B), subsequently moving towards the future ophthalmic lobe of the trigeminal ganglion. The ectoderm becomes devoid of Pax-3 expression at about the 35 -somite stage as trigeminal ganglion cells condense and differentiate. The level of Pax-3 expression in the placodal component of the ophthalmic branch remains significantly higher than in the neural crest component throughout late stages of ganglion formation, allowing easy discrimination between the placode-derived and the neural crest-derived component of the ophthalmic lobe in the con-

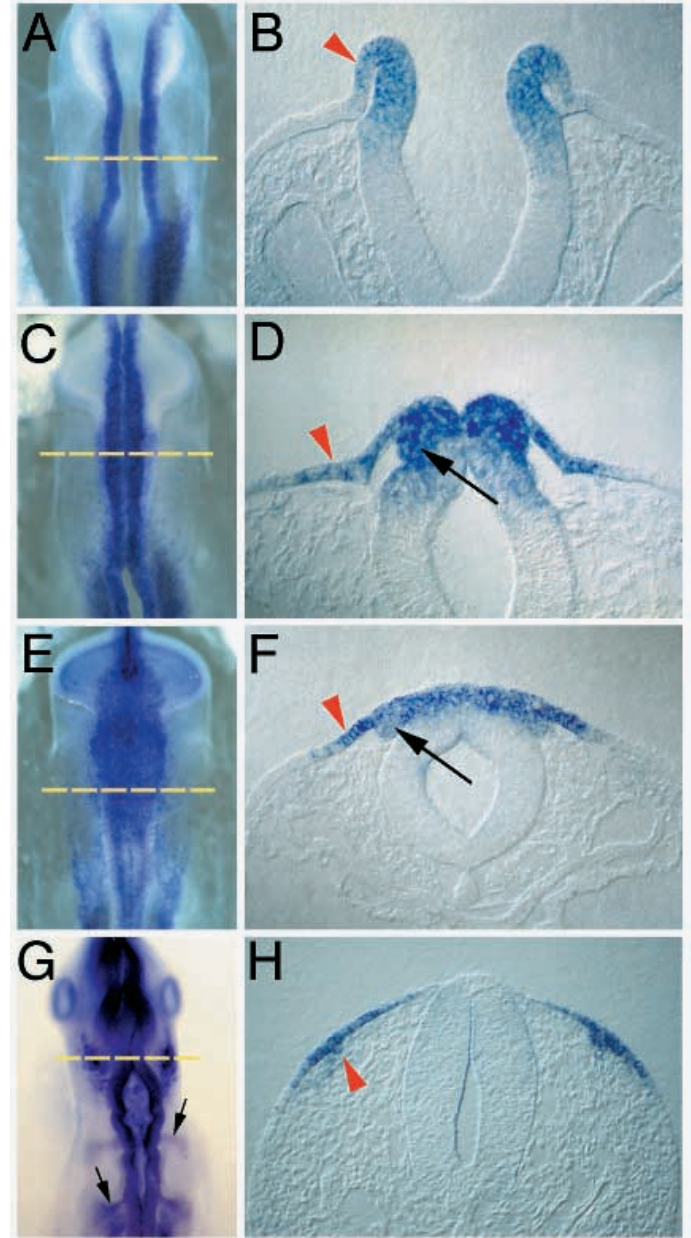

Fig. 3. Expression pattern of Pax-3 in the developing chick ophthalmic lobe placode as revealed by in situ hybridization. (A) A whole-mount view of a 4-somite-stage chick embryo with Pax-3 expression in the dorsal neural folds. (B) A transverse section at the level of the yellow dotted line in A illustrates that Pax-3 expression is confined to the dorsal neural folds and immediately adjacent ectoderm (red arrowhead). (C) A whole-mount view of a 6-somitestage embryo with Pax-3 expression in the newly closed dorsal neural tube and laterally extending ectoderm. (D) A transverse section at the level of the yellow dotted line in C illustrates Pax-3 expression in the dorsal neural tube, prominent in premigratory neural crest cells (arrow) and extending laterally into the adjacent ectoderm (red arrowhead). (E) A whole-mount view of an 8-somitestage chick embryo. (F) A transverse section at the level of the yellow dotted line in E illustrates that Pax-3 expression is largely down-regulated in the neural tube; it is expressed in early migrating neural crest cells (arrow) and prominently in the dorsolateral surface ectoderm (red arrowhead). (G) A whole-mount view of a 16-somitestage chick embryo with Pax-3 expression in opthlamic lobe placode cells. Pax-3 expression is also evident in the neural tube and in streams of migratory neural crest cells rostral and caudal to the otic vesicle (black arrows) $(\mathrm{H}) \mathrm{A}$ transverse section at the level of the yellow dotted line in G shows Pax-3 expression in the lateral ectoderm and in invaginating placode cells (red arrowhead). Note that at this caudal midbrain level, there is no obvious Pax-3 expression in the dorsal neural tube and neural crest.

densing ganglion (Fig. 4C). To determine unequivocally whether Pax-3-positive cells present in the head mesenchyme were derived from the placode, we combined DiI labeling and 
Fig. 4. Trigeminal placode cells in chick as revealed by Pax-3 in situ hybridization. (A) A transverse section through a 13 somite-stage embryo showing invagination of placodal epithelia (red arrowhead). (B) A transverse section through a 20-somite-stage embryo showing a cluster of Pax-3-expressing cells (red arrowhead) which have invaginated into the mesenchyme. (C) A whole-mount view of a stage 17 chick embryo shows Pax-3 expression in the condensing trigeminal ganglion. Pax-3 is highly expressed in the ophthalmic placode-derived portion of the ganglion (red arrowhead), while the neural crest-derived and maxillomandibular placode-derived portions of the ganglion expresses Pax-3 at much lower levels.

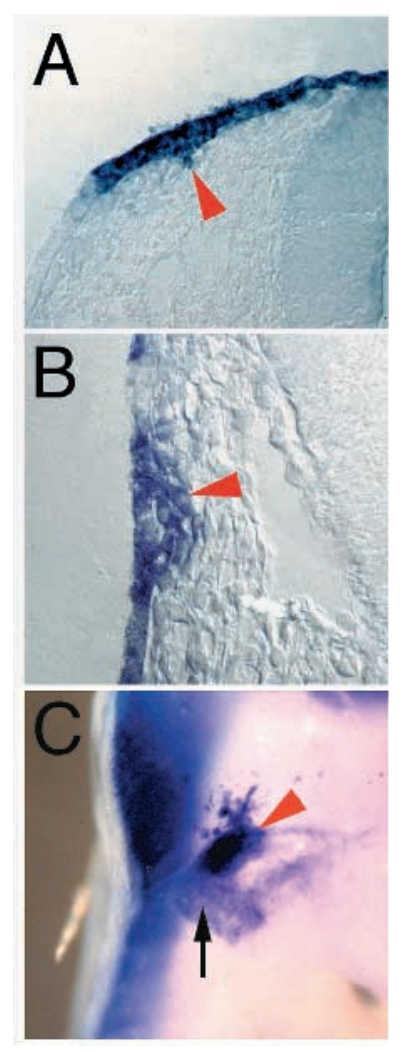

in situ hybridization for Pax-3. We noted numerous DiI-labeled placode cells that were also Pax-3-positive (Fig. 5A-D), confirming that Pax-3-positive cells present in the mesenchyme emanated from the placode.

Conflicting observations have been reported regarding whether or not neural crest cells express Pax-3 (Goulding et al., 1991; Buxton et al., 1997). To reconcile these differences, we carefully examined its pattern of expression during cephalic and trunk crest migration along the body axis; we observed that Pax -3 is only transiently expressed in neural crest cells. While neural folds express Pax-3, its expression appears to be downregulated to almost undetectable transcription levels soon after neural crest cells emigrate from the neural folds and neural tube (Fig. 3F,H). A notable exception is observed at the levels of rhombomeres 4 and 6 , where migrating neural crest cells maintain detectable Pax-3 expression en route to the branchial arches (Fig. 3G). Pax-3 is re-expressed later in embryonic development in the neural crest cells as they condense in dorsal root ganglia (Goulding, 1991; Marcelle et al., 1995) and in some cranial ganglia, including the proximal portion of the ophthalmic lobe as well as the maxillomandibular lobe of the trigeminal ganglion (Fig. 4C). Pax-3 expression in cells contributing to the ophthalmic lobe of the trigeminal ganglion was recently reported (Buxton et al., 1997), although the placodal origin of these cells was not demonstrated. In addition, we noted Pax-3 expression in the neural folds at earlier stages than did Buxton et al. (1997); the differences between our studies are likely due to the different sensitivity of the probes used.

It was previously reported that FREK is expressed in the trigeminal placode (Marcelle et al., 1995). Here, we analyzed the temporal pattern of placodal FREK expression during embryonic development. We found that FREK is expressed in

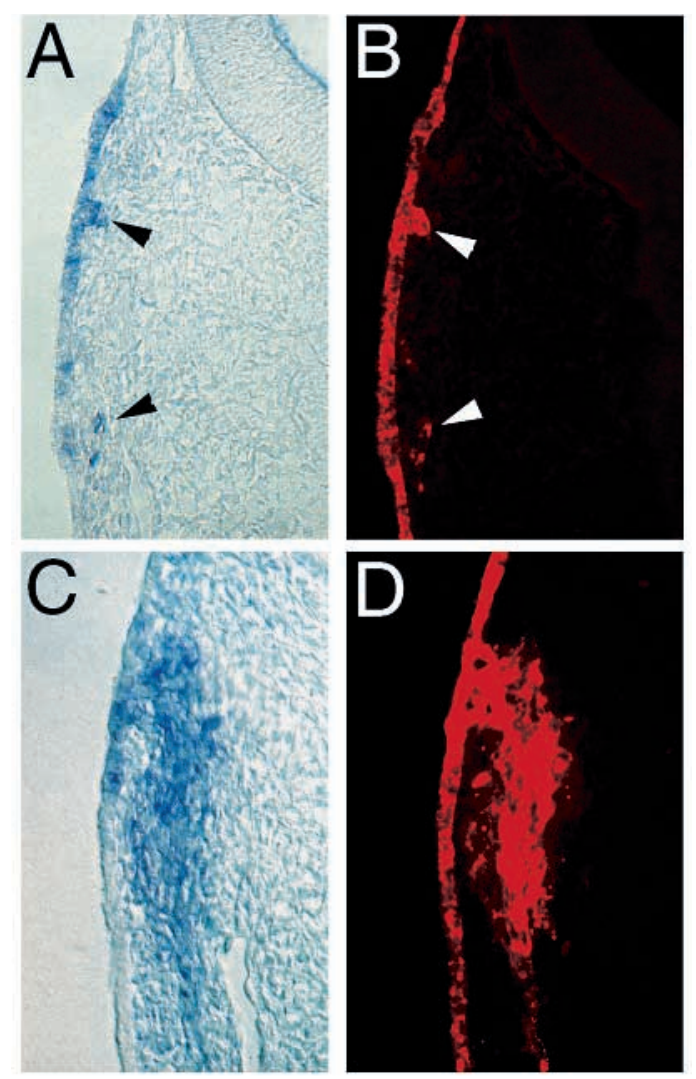

Fig. 5. DiI-labeling of the surface ectoderm confirms that Pax-3 is a molecular marker for invaginating trigeminal placode cells.

(A-D) Coronal sections through the trigeminal placode region of an embryo in which the entire surface ectoderm was labeled with DiI at 12 -somite stage and allowed to develop to 28-somite stage. Bright field $(\mathrm{A}, \mathrm{C})$ shows Pax-3 expression in the ectoderm and in invaginating placode cells. Epifluorescence view $(B, D)$ of the same sections reveals that the invaginated Pax-3-positive cells are also DiI-labeled, confirming their ectodermal origin. Arrowheads (A,B) indicate ophthalmic placode-derived cells.

the ophthalmic branch of the trigeminal placode later than Pax3, with the first FREK-positive cells detectable at the 10-somite stage. Robust FREK expression was observed between the 15to 30-somite stage (Fig. 6A,B) at which time its expression was restricted to ophthalmic lobe placode cells within the ectoderm and to the underlying mesenchyme, similar to the pattern of Pax-3 expression. Unlike Pax-3, FREK expression was not maintained after gangliogenesis.

\section{Pax-3 expression during mouse trigeminal ganglion formation}

It was previously observed that Splotch mice, which carry a non-functional Pax-3 gene, display a reduced ophthalmic lobe of the trigeminal ganglion (Tremblay et al., 1995), suggesting a failure in normal ganglion formation. Pax-3 expression in the mouse trigeminal placode had not been previously reported and this developmental defect was attributed to the neural crest component of the trigeminal ganglion. However, it was recently shown that cephalic neural crest migration and contribution to the trigeminal ganglion appears normal in Splotch mutant mice (Serbedzija and McMahon, 1997). To test whether Pax-3 is expressed in the mouse trigeminal placode, we 


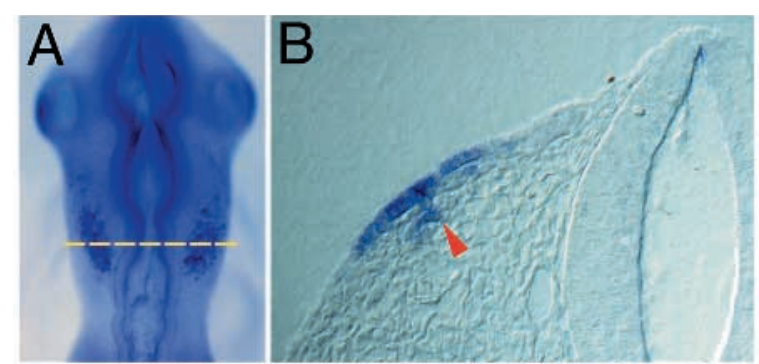

Fig. 6. FREK expression in the trigeminal placode as revealed by in situ hybridization. (A) Whole-mount view of a 16-somite-stage chick embryo with FREK expression in the opthlamic lobe of the trigeminal placode. (B) A transverse section at the level of the yellow dotted line in A shows FREK expression in the lateral ectoderm and in invaginating placode cells (red arrowhead).

performed whole-mount in situ hybridization on 7- to 10-dayold mouse embryos using a mouse Pax-3 probe. Sections through the head show a strong Pax-3 expression domain in the ectoderm adjacent to the midbrain neural folds (Fig. 7A,B) in a region reminiscent of the chick trigeminal placode. These results suggest that, as in chick, the mouse trigeminal placode is expressing Pax-3 and that its expression might be important for placode differentiation.

\section{Neural fold ablation does not alter the placodal expression of Pax-3}

The proximity of neural crest cells to the trigeminal placode raised the possibility that the neural crest may play a role in the induction, migration and/or differentiation of the trigeminal placode. Previous experiments have demonstrated that placode-derived ganglia form when the neural crest component is missing or reduced (Hamburger, 1961); however, these ablations were performed at stage 11 or later, which is after placodal induction and the initiation of neural crest migration. To test whether the presence of the neural crest is required for ophthalmic placode induction, we ablated the dorsal neural folds in the midbrain/hindbrain region either unilaterally or bilaterally in 4- to 8-somite-stage quail and chick embryos. This manipulation effectively removes all neural crest precursors present in the neuroectoderm. The majority of ablations were performed in 6- to 8-somite-stage embryos and collected 6 to 8 hours postsurgery, a time at which there is little or no regeneration of the neural crest (Sechrist et al., 1995); by this

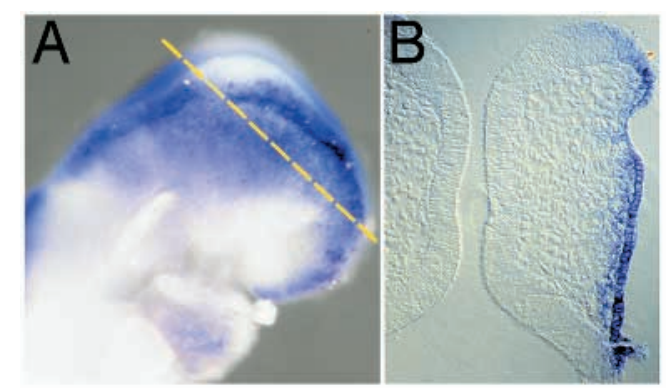

Fig. 7. Pax-3 surface ectoderm expression in the mouse. (A) Wholemount view of a 10-somite mouse embryo showing Pax-3 expression in the dorsal neural tube and the adjacent ectoderm in the head. (B) A transverse section at the level of the yellow dotted line in A shows Pax-3 expression in presumptive placodal ectoderm.

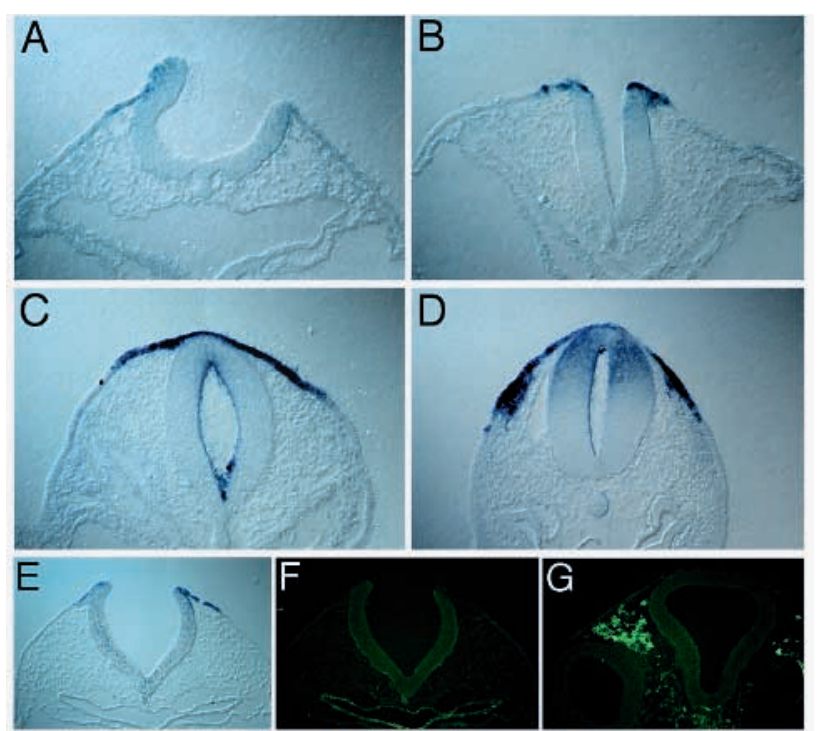

Fig. 8. Effects of unilateral or bilateral neural fold ablation on ectodermal expression of Pax-3. (A) A transverse section through a quail embryo 3 hours after unilateral ablation of the right neural fold at the 4-somite stage. No obvious Pax-3 expression was observed on the operated side. (B) A transverse section through an embryo 4 hours after bilateral neural fold ablation at the 5-somite stage. Pax-3 is expressed in the ectoderm immediately adjacent to the dorsal neural tube. (C) A transverse section through an embryo that underwent bilateral neural fold ablation at the 3-somite stage and was allowed to develop to the 12- to 13-somite stage. Pax-3 is highly expressed in the dorsal ectoderm. (D) A transverse section through an embryo after right unilateral neural fold ablation performed at 5somite stage and fixed at the 19-somite stage; placodal Pax-3 expression was somewhat reduced on the ablated side with fewer cells entering the mesenchyme. However, the overall morphology was similar to that in unoperated embryos. (E) Transverse section through the caudal midbrain of an 11-somite embryo that underwent bilateral neural fold ablation at the 6-somite stage and was allowed to develop 9 hours to the 11-somite stage. Although neural tube closure has not yet occurred, Pax-3 is expressed in the surface ectoderm. (F) Fluorescent image of the same section after staining with the neural crest antibody HNK-1. Neural crest cells were absent at this axial level. (G) Transverse section illustrating the presence of numerous HNK-1 immunoreactive neural crest cells at a more rostral region (adjacent to the caudal portion of the optic cup) of the same embryo shown in $\mathrm{E}$ and $\mathrm{F}$.

stage, Pax-3 expression had presumably expanded beyond the lateral margins of the neural folds (Fig. 3C,D), in which case we also removed any putative Pax-3-expressing surface ectoderm. Embryos were collected from 1 to 48 hours after ablation and evaluated for placode induction and ganglion formation by in situ hybridization with the Pax-3 probe.

From 0 to 3 hours postablation ( $n=4), P a x-3$ was absent in the ectoderm adjacent to the neural tube shortly after ablation (Fig. 8A), indicating effective removal of both the neural crest and placodal precursors. However, in all embryos fixed between 4 and 9 hours postablation $(n=16)$, some ectodermal Pax-3 expression was observed, with the level of expression dependent upon the degree of healing between the neuroectoderm and adjacent surface ectoderm (Fig. 8B). By 11 or more hours postsurgery (n=8), Pax-3-positive ectodermal cells could be detected immediately adjacent to the neuroepithelium (Fig. 
8C). After 16 to 24 hours, all embryos showed Pax-3 expression in both the ectoderm and invaginating placode cells (Fig. 8D). The number of Pax-3-expressing placode cells and their intensity of staining varied depending on the depth of ablation and degree of healing. However, the complement of cells was generally normal or only slightly reduced. Pax-3 expression in the dorsal neural tube also resumed in these embryos by this stage. Embryos collected after gangliogenesis often had trigeminal ganglia that appeared misshapen and improperly located. Staining with the HNK-1 antibody, which recognizes migrating neural crest cells, confirmed the absence of neural crest cells in the region of the ablation in embryos collected 6 to 8 hours postsurgery (Fig. 8E-G). In several embryos, placodal Pax-3 expression was observed in the absence of midline closure, which has recently been suggested to be necessary for Slug expression and neural crest formation (Buxton et al., 1997). The observation that Pax-3 is expressed in the placodes soon after neural fold ablation and prior to the appearance of neural crest cells suggests that induction and/or maintenance of the ophthalmic lobe placode is independent of the neural folds/neural crest.

The experiments described above were repeated using DiIlabeling to mark the surface ectoderm after neural crest or surface ectoderm ablation. Following neural crest ablation at the 4- to 7-somite stage, chick embryos were allowed to heal and develop to the 15- to 20-somite stage, at which time DiI was applied. Embryos were allowed to develop for an additional 48 hours. In all cases, placode-derived ganglia developed in the region of the trigeminal system, although most were displaced and misshapened without an apparent neural crest component (Fig. 9A). This result confirms that obtained with Pax-3 and indicates that placodal cells can invaginate in the absence of neural crest cells. To remove the trigeminal placode, embryos underwent ectoderm ablation larger than the presumptive trigeminal placode at the 12-somite stage and were allowed to heal and develop to the 18- to 20-somite stage prior to DiI surface ectoderm labeling. 1 day later, the placodederived component of the ganglion was reduced but never absent (Fig. 9B). Our inability to completely remove the placode by ablation is likely to be due to the ability of the surface ectoderm to efficiently regenerate placodal epithelium, as suggested by Hamburger (1961).

\section{A diffusible signal from the neural tube is required for normal placode formation}

To test whether neuroectoderm-ectoderm interactions are necessary for proper trigeminal placode formation, we surgically separated the ectoderm from the neural folds in vivo in 2- to 9-somite-stage embryos and prevented subsequent reclosure and contact by placing an impermeable foil barrier between the two tissues (Fig. 10A,B). In most experiments $(n=20)$, a $7.5 \mu \mathrm{m}$ thick tantalum foil barrier was used, although we found that gold $(n=4)$ or aluminium $(n=6)$ foil barriers were equally effective in preventing neuroectoderm-ectoderm contact with no apparent toxic or teratogenic effects.

Blocking ectoderm-neural tube interactions led to a reduction or complete loss of Pax-3 expression in the surface ectoderm (Fig. 10A,B). The extent of placode loss varied with the length of the barrier, its rostrocaudal location and the stage of barrier implantation. For example, barriers placed between the neural tube and the entire length of the presumptive oph-

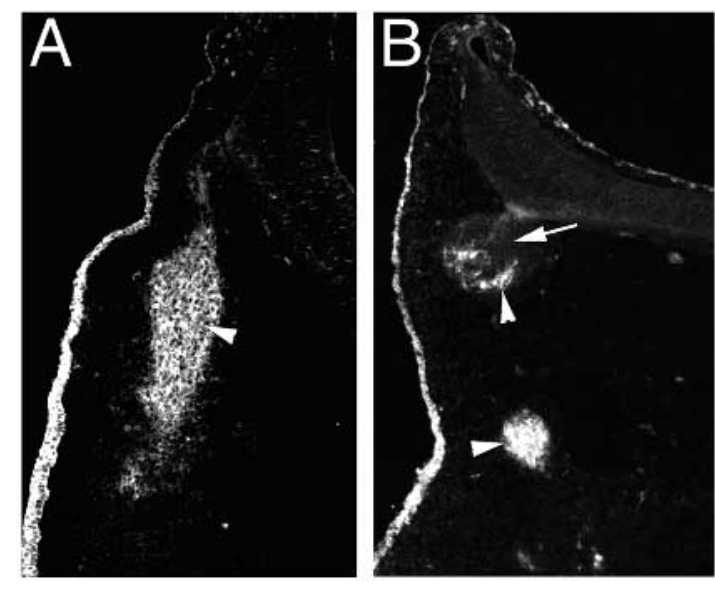

Fig. 9. Effects of neural crest or placode ablation on ganglion formation as revealed by surface ectoderm DiI labeling. (A) Section through a 33-somite-stage embryo which underwent neural fold ablation at the 5-somite stage and was labeled with DiI at the 18somite stage. The neural crest component of the trigeminal ganglion is largely absent while the DiI-labeled placode component comprises the remaining ganglion (arrowhead). (B) Section through a 35somite-stage embryo which underwent ablation of the presumptive placodal ectoderm at the 12-somite stage and was labeled following healing at the 19-somite stage. The neural crest component of the ganglion appears intact (arrow) while the DiI-labeled placode cells are reduced in number with the majority of cells having condensed external to the ganglion proper (arrowhead). Compare with section through an unablated embryo shown in Fig. 2D.

thalmic placode reduced the levels of Pax-3 expression by 70$100 \%(n=19)$. Barriers that were smaller or implanted more caudally or rostrally resulted in a 30-70\% reduction in Pax-3 expression $(n=4)$. In several cases $(n=9)$, the neural folds were ablated unilaterally or bilaterally prior to barrier implantation; no obvious differences in the amount of Pax-3 loss lateral to the barrier were observed in the presence or absence of the neural folds. Prior to and shortly after invaginating from the ectoderm, some placodal cells begin to express neuronal differentiation markers such as $\beta$-tubulin (Moody et al., 1989) and become neurofilament immunoreactive (unpublished observation). Implantation of impermeable barriers $(n=8)$ resulted in a loss or profound reduction in neurofilament immunoreactivity on the operated side of the embryo (Fig. 10C), confirming that the loss of Pax-3 expression correlated with the loss of normal differentiation of ophthalmic placode cells. As a control $(n=4)$, barriers were removed shortly after insertion; in these embryos, placodal Pax-3 $(n=3)$ or neurofilament $(n=1)$ expression appeared normal. These results indicate that a neuroectodermectoderm interaction is required for normal placode induction and/or maintenance.

The ectoderm could receive signals from the neural tube either by means of cell contact or diffusible molecules. As a first step in determining the molecular nature of underlying trigeminal placode induction and/or maintenance), we placed polycarbonate barriers (Schramm et al., 1994) with pore sizes of $0.1 \mu \mathrm{m}$ (allowing passage of diffusible molecules; $n=12$ ) or $0.8 \mu \mathrm{m}$ (allowing passage of both cell processes and diffusible molecules; $n=11$ ) between the ectoderm and neural tube of 4to 9-somite-stage chick embryos. Pax-3 expression was observed in the ectoderm overlying either large pore size 
Fig. 10. Pax-3 expression $(A, B)$ or neurofillament immunoreactivity (C) in embryos after implantation of impermeable barriers (represented by dotted lines in B,C). (A,B) An embryo into which a tantalum foil barrier was placed between the neural tube and ectoderm at the 6somite stage and subsequently allowed to develop for 24 hours. Placodal Pax-3 expression was
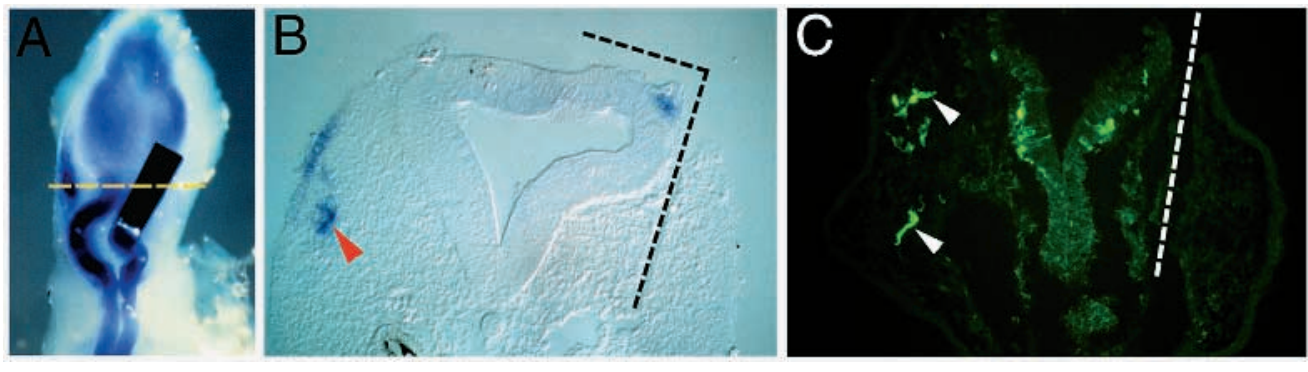

eliminated on the operated side, while expression on the control side was unaffected. (C) Section through an embryo into which a tantalum foil barrier was placed between the neural tube and ectoderm at the 6-somite stage and subsequently allowed to develop for 24 hours.

Neurofilament-positive cells were absent from the operated side, whereas the control side had a normal complement of neurofilament-positive placode cells (arrowheads).

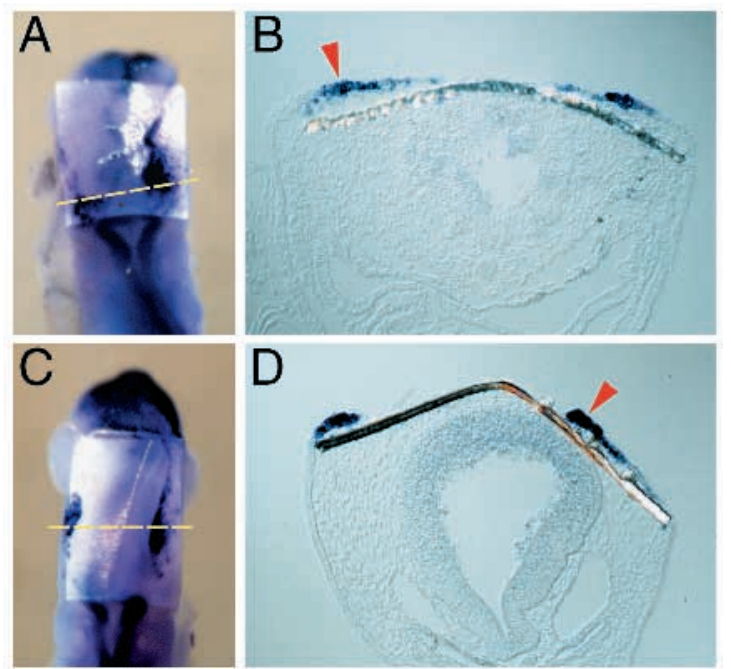

Fig. 11. Pax-3 expression in embryos after implantation of semipermeable barriers. Placodal Pax-3 expression after implantation of a $0.8(\mathrm{~A}, \mathrm{~B})$ or $0.1(\mathrm{C}, \mathrm{D}) \mu \mathrm{m}$ polycarbonate barriers between the neural tube and ectoderm. Surgeries were performed at 6- to 7-somite stage and the embryo was allowed to develop for an additional 24 hours. Pax-3 is expressed in the ectoderm in the presence of either pore size barrier.

barriers (Fig. 11A,B) or small pore size barriers (Fig. 11C,D), indicating that a diffusible signal from the neural tube is sufficient to induce and/or maintain expression of $\mathrm{Pax}_{-3} 3$ in the trigeminal placode.

\section{DISCUSSION}

Due to the lack of appropriate molecular markers, little was known about the early inductive events leading to placode formation and differentiation. We report that Pax-3 and FREK are expressed in placode cells contributing to the ophthalmic lobe of the trigeminal ganglion, from the time of early specification (Pax-3 and FREK) through ganglion formation (Pax-3 only). Analysis of Pax-3 expression indicates that placode cells are specified early in development. We observed Pax-3 expression as early as the 4-somite stage, 10 to 15 hours before placode cells initiate migration towards the future ganglion. Chick/quail chimeric studies have suggested that the trigeminal placodes originate in the neural folds and subsequently translocate ventrolaterally through the ectodermal layer (Noden, 1983; D'Amico-Martel and Noden, 1983; Couly and Le Douarin, 1990). However, we find that the presence of a permeable, physical barrier between the neuroectoderm and the surface ectoderm does not prevent Pax-3 expression and subsequent ganglion formation, despite the fact that it would be expected to block lateral migration; rather than arising by migration from the neural folds, our data suggest that the ophthalmic placode is induced within the surface ectoderm by a diffusible signal from the neuroectoderm.

Although our experiments utilize Pax-3 solely as a molecular marker for trigeminal placode cells, Pax-3 is also likely to play a functional role in ganglion formation. The conservation of the distribution patterns of Pax-3 between the chick and mouse trigeminal placode is consistent with the possibility that $P a x-3$ is necessary for proper trigeminal ganglion formation. Furthermore, Splotch mutants that are deficient in Pax-3 have significant reductions in the ophthalmic lobe of the trigeminal ganglion (Tremblay et al., 1995). In addition, these mice display major defects in skeletal muscle progenitor migration towards the limb mesenchyme while muscle differentiation is unimpaired (Datson et al., 1996). Like muscle progenitors of the lateral somite, placode cells express high levels of Pax-3 prior to undergoing an epithelial-mesenchymal transition and initiating migration. One intriguing possibility is that Pax-3 may be important for placode cell migration toward the condensing ganglion within the head mesenchyme. Another interesting parallel between skeletal muscle precursors and trigeminal placode cells is that they both express the fibroblast growth factor receptor FREK following expression of Pax-3 (Marcelle et al., 1995). This raises the possibility that this receptor may be a target of the transcription factor.

Interestingly, Pax-3 and FREK are markers of the ophthalmic lobe placode, while the maxillomandibular lobe placode is devoid of their expression. Morphological studies performed in amphibians have suggested that the ophthalmic and the mandibular lobes of the trigeminal ganglion are embryologically and evolutionarily distinct (reviewed by Hamburger, 1961; Northcutt and Brandle, 1995). Our observation that Pax3 and FREK are initially expressed in the ophthalmic and not in the mandibular branch of the ganglion is consistent with this hypothesis, since these two branches of the ganglion are molecularly distinct from one other.

The cranial ganglia have a dual origin from neural crest cells and sensory ectodermal placodes (reviewed in Le Douarin et al., 1986; Webb and Noden, 1993). Ectodermal placode cells share 
many properties with neural crest cells including the ability to undergo an epithelial-to-mesenchymal transition, migrate and contribute to neuronal components of sensory ganglia. The proximity of neural crest cells to the trigeminal placode raised the prospect that the neural crest could play a role in the induction, migration and/or differentiation of the trigeminal placode. Our results, however, rule out this possibility since Pax-3 expression and ophthalmic lobe formation can occur in the absence of neural crest cells. The placode-derived trigeminal ganglion of neural-fold-ablated embryos appeared misshapen and improperly located, however, suggesting that the normal complement of neural crest cells may at least play a role in the organization and position of the ganglion. Hamburger (1961) previously concluded that placode cell differentiation was independent of neural crest cells; these experiments, however, were performed in 12- to 20-somite-stage embryos, after initiation of neural crest migration and placodal specification, as established by the present results.

Previous work from our laboratory has shown that neural crest cells regenerate after ablation of the dorsal neural folds (Scherson et al., 1993; Sechrist et al., 1995). However, optimal regeneration occurs when neural folds are ablated at or prior to the 4-somite stage whereas, in the present study, the majority of ablations were performed after the time of optimal regeneration. Furthermore, production of neural crest cells after ablation is delayed compared with initial generation of neural crest cells, with the first regenerated HNK-1-positive cells observed at $\sim 13$-somite stage (Sechrist et al., 1995). In the present study, we observe placodal Pax-3 expression as early as 4 hours postablation, prior to any detectable neural crest regeneration. Furthermore, Buxton et al. (1997) have suggested that midline closure is necessary for re-expression of the neural crest marker Slug after neural fold ablation. Because we observe placodal Pax-3 expression after ablation but prior to dorsal midline closure, our results demonstrate that neural crest cells are not required for induction or maintenance of Pax-3 in the placodal ectoderm.

Previous studies have shown that neural crest cells can form via an inductive interaction between neural tissue and nonneuronal ectoderm (Moury and Jacobson, 1989; Selleck and Bronner-Fraser, 1995; Dickinson et al., 1995). Dorsalin-1, BMP-4 and BMP-7 have been shown to be sufficient to substitute for the non-neural ectoderm in inducing neural crest cells (Basler et al., 1993; Liem et al., 1995). In contrast, the inductive interactions necessary for placode formation have been elusive. There is some evidence that the otic placode may be induced by the adjacent hindbrain (Waddington, 1937; Sechrist et al., 1994) although putative inducers have not been identified (McKay et al., 1996). In this study, we show that, similar to the neural crest, the ophthalmic lobe placode may arise from a neuroectoderm-ectoderm interaction. A signal emanating from the neural tube is required for placode formation and neuronal differentiation, as assayed by Pax-3 expression in the placode and by neurofilament expression. Members of the TGF $\beta$ and Wnt families are expressed in the head neuroectoderm at developmental stages compatible with a putative role in trigeminal placode induction. Future experiments will test the role of various candidate inducers in the formation of the trigeminal placode and examine whether similar interactions are responsible for the formation of other ectodermal placodes.
We thank Angela Nieto for early contributions to this work and Martin Goulding for the chick and mouse Pax-3 probe. We are indebted to Brian Rowe, Roham Zamanian, Johnny Choi, and Parisa Zarbafian for technical assistance and Drs Clare Baker, Andrew Groves, Catherine Krull and Ben Murray for critical reading of the manuscript. This work was supported by NS34671 to M. B. F. and a grant from the Muscular Dystrophy Association to C. M.

\section{REFERENCES}

Ayer-Le Lièvre, C. S. and Le Douarin, N. M. (1982). The early development of cranial sensory ganglia and the potentialities of their component cells studied in quail-chick chimeras. Dev. Biol. 94, 291-310.

Basler, K., Edlund, T., Jessell, T. M. and Yamada, T. (1993). Control of cell pattern in the neural tube: Regulation of cell differentiation by dorsalin-1, a novel TGF $\beta$ family member. Cell 73, 687-702.

Buxton, P., Hunt, P., Ferretti, P. and Thorogood, P. (1997). A role for midline closure in the reestablishment of dorsoventral pattern following dorsal hindbrain ablation. Dev. Biol. 183, 150-165.

Chalepakis, G., Stoykova, A., Wijnholds, J., Tremblay, P. and Gruss, P. (1993). Pax: gene regulators in the developing nervous system. J. Neurobiol. 24, 1367-1384.

Couly, G. and Le Douarin, N. M. (1990). Head morphogenesis in embryonic avian chimeras: evidence for a segmental pattern in the ectoderm corresponding to the neuromeres. Development 108, 543-558.

Datson, G., Lamar, E., Olivier, M. and Goulding, M. (1996). Pax-3 is necessary for migration but not differentiation of limb muscle precursors in the mouse. Development 122, 1017-1027.

D' Amico-Martel, A. and Noden, D. M. (1980). An autoradiographic analysis of the development of the chick trigeminal ganglion. J. Embryol. Exp. Morph. 55, 167-182.

D’Amico-Martel, A. and Noden, D. M. (1983). Contributions of placodal and neural crest cells to avian cranial peripheral ganglia. Am. J. Anat. 66, 445468.

Davies, A. M. (1988). The trigeminal system: an advantageous experimental model for studying neuronal development. Development 103 Supplement, 175-183.

Dickinson, M., Selleck, M., Mcmahon, A. and Bronner-Fraser, M. (1995). Dosalization of the neural tube by the non-neural ectoderm. Development 121, 2099-2106.

Goulding, M. D., Chalepakis, G., Deutsch, U., Erselius, J. and Gruss, P. (1991). Pax-3, a novel murine DNA binding protein expressed during early neurogenesis. EMBO J. 10, 1135-1147.

Goulding, M. D., Lumsden, A. and Gruss, P. (1993). Signals from the notochord and floor plate regulate the region specific expression of two Pax genes in the developing spinal cord. Development 117, 1001-1016.

Hamburger, V. (1961). Experimental analysis of the dual origin of the trigeminal ganglion in the chick embryo. J. Exp. Zool. 148, 91-124.

Henrique, D., Adam, J., Myat, A., Chitnis, A., Lewis, J., Ish-Horowicz, D. (1995). Expression of a Delta homologue in prospective neurons in the chick. Nature 375, 787-790.

Kuratani, S. C. and Hirano, S. (1990). The appearance of trigeminal ectopic ganglia within the surface ectoderm in the chick embryo. Arch. Histol. Cytol. $53,575-583$.

Le Douarin, N. M. (1982). The Neural Crest. London: Cambridge University Press.

Le Douarin, N. M., Fontaine-Pérus, J. and Couly, G. (1986). Cephalic ectodermal placodes and neurogenesis. Trends Neurosci. 9, 175-180.

Liem, K. F., Tremml, G., Roelink, H., and Jessell, T. M. (1995). Dorsal differentiation of neural plate cells induced by BMP-mediated signals from epidermal ectoderm. Cell 82, 969-979.

Marcelle, C., Eichmann, A., Halevy, O., Bréant, C. and Le Douarin, N. M. (1994). Distinct developmental expression of a new avian fibroblast growth factor receptor. Development 120, 683-694.

Marcelle, C., Wolf, J. and Bronner-Fraser, M. (1995). The in vivo expression of the FGF receptor FREK mRNA in avian myoblasts suggests a role in muscle growth and differentiation. Dev. Biol. 172, 100-114.

McKay, I. J., Lewis, J., and Lumsden, A. (1996). The role of FGF-3 in early inner ear development: an analysis in normal and kreisler mutant mice. Dev. Biol. 174, 370-378.

Moody, S. A., Quigg, M. S. and Frankfurter, A. (1989). Development of the peripheral trigeminal system in the chick revealed by an isotype-specific antibeta-tubulin monoclonal antibody. J. Comp. Neurol. 279, 567-580. 
Moury, J. D. and Jacobson, A. G. (1989). Neural fold formation at newly created boundaries between neural plate and epidermis in the axolotl. Dev Biol. 133, 44. 57.

Narayanan, C. H. and Narayanan, Y. (1980). Neural crest and placodal contributions in the development of the glossopharyngeal-vagal complex in the chick. Anat. Rec. 196, 71-82.

Nichols, D. H. (1986). Mesenchyme formation from the trigeminal placodes of the mouse embryo. Am. J. Anat. 176, 19-31.

Noden, D. M. (1978). The control of avian cephalic neural crest cytodifferentiation. II. Neural Tissues. Dev. Biol. 67, 313-329.

Noden, D. M. (1983). The role of the neural crest in patterning of avian cranial skeletal, connective, and muscle tissues. Dev. Biol. 96, 144-165.

Noden, D. M. (1993). Spatial integration among cells forming the cranial peripheral nervous system. J. Neurobiol. 24, 248-261.

Northcutt, R. G. and Brandle, K. (1995). Development of branchiomeric and lateral line nerves in the axolotl. J. Comp. Neurol. 355, 427-454.

Sanger, F., Nicklen, S. and Coulson, A. R. (1977). DNA sequencing with chain termination inhibitors. Proc. Natl. Acad. Sci. USA 74, 5463-5467.

Scherson, T., Serbedzija, G., Fraser, S. and Bronner-Fraser, M. (1993). Regulative capacity of the cranial neural tube to form neural crest. Development 118, 1049-1061.

Schramm, C. A., Reiter, R. S. and Solursh, M. (1994). Role for short-range interactions in the formation of cartilage and muscle masses in transfilter micromass cultures. Dev. Biol. 163, 467-479 (1994).

Sechrist, J., Serbedzija, G., Scherson, T., Fraser, S., and Bronner-Fraser, M. (1993). Segmental migration of the hindbrain neural crest does not arise from segmental generation. Development 118, 691-703.
Sechrist, J., Scherson, T. and Bronner-Fraser, M. (1994). Rhombomere rotation reveals that multiple mechanisms contribute to the segmental pattern of hindbrain neural crest migration. Development 120, 1777-1790.

Sechrist, J., Nieto, M. A., Zamanian, R. T. and Bronner-Fraser, M. (1995). Regulative response of the cranial neural tube after neural fold ablation: spatiotemporal nature of neural crest regeneration and up-regulation of Slug. Development 121, 4103-4115.

Selleck, M. A. and Bronner-Fraser, M. (1995). Origins of the avian neural crest: the role of neural plate-epidermal interactions. Development 121, 525 538

Serbedzija, G. and McMahon, A. P. (1997). Analysis of neural crest cell migration in Splotch mice using a neural crest-specific lacZ reporter. Dev. Biol. 185, 139-147.

Tremblay, P., Kessel, M. and Gruss, P. (1995). A transgenic neuraoanatomical marker identifies cranial neural crest deficiencies associated with the Pax-3 mutant Splotch. Dev. Bio.l 171, 317-329.

Waddington, C. H. (1937). The determination of the auditory placode in the chick. J. Exp. Biol. 14, 232-239.

Webb, J. W. and Noden, D. M. (1993). Ectodermal placodes: contributions to the development of the vertebrate head. Amer. Zool. 33, 434-447.

Wilkinson, D. G. (1992). Whole mount in situ hybridization of vertebrate embryos. In In Situ Hybridization (ed. D. G. Wilkinson). IRL Press, Oxford.

Yntema, C. L. (1942). Experiments on the origin of some of the sensory cranial ganglia in the chick. Anat. Rec. 82, 455. 